\title{
External Iliac Vein Laceration-A Rare Complication of Tunneled Dialysis Catheter
}

\author{
Hashmi MN*, Rashid Asghar, Tanzeel Abbasi, Khurram Bashir and Wajahat Ali \\ Multan Institute of Kidney disease, Multan, Pakistan
}

*Corresponding author: Hashmi MN, Multan Institute of Kidney disease,

Multan, Pakistan.

Received Date: August 12,2020

Published Date: August 31,2020

\begin{abstract}
Bleeding complication from Central dialysis catheter is rare. Usually it settles in short time after procedure. If patient is on antiplatelet therapy it can sometimes last for 24-48 hours and stops with pressure dressing. We report a rare case of ongoing ( $>96$ hour post procedure) bleeding from dialysis catheter exit site and investigation revealed the cause was iliac vein laceration.
\end{abstract}

\section{Introduction}

Vascular access is life line of hemodialysis patients. Preferred choice is AVF but sometimes while awaiting maturation of AVF, a tunneled dialysis catheter is placed to fulfill need of renal replacement therapy. Dialysis catheter placement can sometimes result in complications and they are dealt accordingly.

\section{Case}

56-year-old female patient on Maintenance Hemodialysis underwent a new arteriovenous fistula (AVF) creation. She also underwent right tunneled femoral dialysis catheter insertion to cover dialysis till fistula is matured. She presented to OPD with bleeding at site of Catheter insertion during hemodialysis. This is common in newly inserted catheter especially if patient is on antiplatelet therapy. It usually stops in 24-48 hrs. Patient was examined and no obvious hematoma or bleeding cause identified at insertion site of femoral dialysis catheter. Exit site was clean (no evidence of infection) and all stitches were removed. Patient's drug history was reviewed and she was not on any anticoagulation therapy. Laboratory parameters showed Normal coagulation profile, Hemoglobin 8.6g/dl and platelet count of 235×109/L. Patient later went for hemodialysis and on commencement of dialysis bleeding started from insertion site of dialysis catheter. This was unusual and in order to identify the cause we discussed case with radiology department and CT fluoroscopy was planned. Patient underwent CT to detect bleeding point. She had CT fluoroscopic angiogram (160 slice strength). Ultravist-300 Contrast medium of $100 \mathrm{ml}$ was injected in femoral dialysis catheter. Imaging showed extra vasation of the contrast medium from external iliac vein at the level of femoral head. Laceration was $8 \mathrm{~cm}$ away from insertion point of catheter (Figure 1,2).

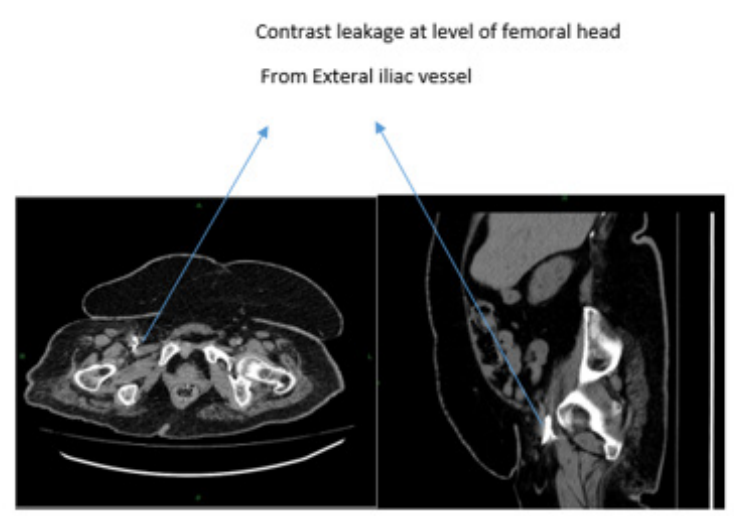

Figure 1 \& 2: Axial and Sagittal Section of CT fluoroscopy. 


\section{Discussion}

Maintenance Hemodialysis patients lifeline is vascular access. Vascular access patency is another crucial step. AVF is preferred choice in patients who can tolerate surgery. Pre-emptive AVF is preferred choice [1]. A lot of patients had to undergo Permanent catheter insertion as initial access for bridging AVF maturation. Central Venous catheters (CVC) insertion complications include vascular injury (arterial puncture, pseudoaneurysm, air embolism, pneumothorax, and malposition). Indwelling complications are infection, thrombosis, catheter pinching/kinking, and fracture with possible embolization. Late complication is stenosis of veins that may occur over a period of time, after damage to the vein wall due to infection or mechanical stress. The risk of stenosis is reduced if the catheter lies in the center of a big vein with a high blood flow away from junctions with other veins [2]. Approximately one-third of hemodialysis patients who use tunneled dialysis catheter during 1 to 2 years' experience complications [3]. Central venous stenosis has been reported in studies and usually occurs in small number of patients but more common in patients who undergo repeated CVC insertion or with long duration of catheter [4]. Our patient had bleeding secondary to iliac vein damage at level of femoral head anatomically which is rare. Patient had history of access issues and had multiple temporary lines insertion in past resulting in stenosis. In our case catheter placement was done by interventional radiologist and we suspect due to previous history of multiple dialysis lines placement there was stenosis present. On passing dilator the vein got damaged and resulted in iliac vein laceration. There is also possibility of vein damage from catheter tip which has been reported in case report [5]. Patient was referred to vascular surgery after confirmation of vein laceration and the injured vessel wall was treated surgically. Patient has been well since then and undergoing regular hemodialysis from AVF.

\section{Conclusion}

We wish to point rare complication of hemodialysis catheter which can happen during catheter placement. In such cases early investigation can reduce complications. Our patient was timely diagnosed and treated.

\section{Acknowledgments}

None.

\section{Conflict of Interest}

No conflict of interest.

\section{References}

1. Arhuidese IJ, Cooper MA, Rizwan M, Nejim B, Malas MB (2019) Vascular access for hemodialysis in the elderly. J Vasc Surg 69(2): 517-525.e1.

2. Santoro D, Benedetto F, Mondello P, Pipito N, Barilla D, et al. (2014) Vascular access for hemodialysis: current perspectives. Int J Nephrol Renovasc Dis 7: 281-294.

3. Poinen K, Quinn RR, Clarke A, Ravani P, Hiremath S, et al. (2019) Complications From Tunneled Hemodialysis Catheters: A Canadian Observational Cohort Study. Am J Kidney Dis 73(4):467-475.

4. Adwaney A, Lim C, Blakey S, Duncan N, Ashby DR (2019) Central Venous Stenosis, Access Outcome and Survival in Patients undergoing Maintenance Hemodialysis. Clin J Am SocNephrol 14(3): 378-384.

5. Stolic, Radojica V, Sanja P Milojevic (2011) Laceration of the iliac vein in a patient with a femoral catheter for hemodialysis. International journal of nephrology 2011: 919858. 